\title{
HACIA UNA CARACTERIZACIÓN SEMÁNTICA DE LA TERMINOLOGÍA LINGÜÍSTICA
}

\author{
Miguel Casas Gómez \\ (Universidad de Cádiz)
}

\begin{abstract}
RESUMEN
The main aims of this article are to undertake a study, based upon an analysis of linguistic terms such as moneme, morpheme, semantics, semasiology, onomasiology, signification, etc., of how certain lexical relationships in this terminological field are presented, and to reflect upon the special semantic characteristics of this technical lexicon in relation to the principles which govern other scientific terminologies.
\end{abstract}

0. La lingüística, al igual que toda ciencia, requiere como condición necesaria para constituirse como tal, además de un objeto y un método, una terminología específica y precisa, la cual, en esta parcela, comporta una dificultad que hemos de superar. El desarrollo en los últimos cien años de las diferentes disciplinas lingüísticas ha provocado tal dispersión terminológica que no parece excesivo afirmar que en la actualidad nos encontramos ante un caos en que sinonimia, polisemia y ambiguiedad contradicen de hecho las finalidades básicas de los metalenguajes científicos, que, dada la función que desempeñan, cual es la de clasificar o delimitar la realidad objetiva de los fenómenos u objetos definidos por las respectivas ciencias o técnicas, presentan generalmente un carácter monosémico. Todos somos conscientes de las confusiones que provoca esta proliferación de términos, que ha exigido la necesaria aparición de un considerable número de diccionarios técnicos especializados ${ }^{1}$, por lo que cada vez con más

1 Entre otros diccionarios y demás obras de carácter lexicográfico, véanse los trabajos de J. Marouzeau (1951); F. Lázaro Carreter (1974); O. Ducrot / T. Todorov (1972); A. Martinet (1972); R. R. K. Hartmann / F. C. Stork (1972); J. Dubois y otros (1973); B. Pottier (1973); G. Mounin (1974); Z. dos S. Jota (1976); A.-J. Greimas / J. Courtés (1979); W. Abraham (1981); T. Lewandowski (1982); W. Welte (1985), y G. R. Cardona (1991). 
urgencia se impone una unificación lo más amplia posible de esta terminología científica.

Representativa a este respecto fue la propuesta que, a finales de los años setenta, hiciera el lingüista pragense $O$. Duchácek (1979: 10-11 y 17-19), con objeto de llegar a una normalización de las terminologías que reservara el empleo de términos actualmente sinónimos para distinguir nociones estrechamente emparentadas (cf. n. 13) y suprimiera, de este modo, la sinonimia inútil. Pero estos esfuerzos, en su opinión, están abocados al fracaso si no vienen refrendados por una institución que tenga la competencia de decretar una terminología oficial que todo especialista deberá aceptar. No obstante, este problema ya fue debatido en 1948 durante el VI Congreso Internacional de Lingüistas y de él se hace eco también S. Ullmann (1952: 209-210; 1957: 121, y 1964a: 171-172), que pone de manifiesto cómo la ambigüedad en el uso técnico-científico, complicada en ciertas ocasiones con hechos de sinonimia (cf. n. 4), constituye uno de los tipos de situaciones susceptibles de producir una «polisemia patológica». Entre los equívocos específicos del ámbito lingüístico, cita los casos de estilística, fonema, morfema, semántica, significación, significado, oración ${ }^{2}$, estructuralismo y fonología ${ }^{3}$, y alude con ello a la propuesta - sugerida en el marco de la psicología - de dotar a los términos de un subíndice, del tipo funcionalismo ${ }_{\text {Coseriu }}$ que aclare el sentido en que los empleamos. Añade, además, que estas confusiones se intensifican cuando palabras usadas de forma ambigua en la lengua común se utilizan igualmente en diferentes contextos técnico-científicos, que requieren la máxima precisión conceptual (así, por ejemplo, causa, ley, verdad, signo, símbolo, etc.). Sin embargo, este autor, a pesar de plantear también la imperiosa necesidad de coordinar y normalizar la ambigüedad inherente a estas terminologias «against which 'general' semanticists have proclaimed a crusade» (1957: 121), se muestra no sólo escéptico ante la posibilidad de tal uniformidad, sino que incluso se pregunta por el inconveniente que supondría la «standardisation rigide» de esta ortodoxia terminológica.

Más modernamente, estos aspectos serán anotados por E. Coseriu (1981a: 124-127) como hechos que contribuyen, en gran parte, a la complejidad y dificultad de la terminología lingüística actual. Así, para los casos en que un mismo término adquiere - según diferentes concepciones teóricas - valores diversos, ejemplifica con el término morfema, del que realiza un análisis detallado de sus respectivos usos técnicos de acuerdo con distintas orientaciones metodológicas. $\mathrm{Y}$ en lo que concierne al empleo de términos cercanos o coincidentes desde el punto de vista material con palabras del léxico usual

2 Léanse las más de doscientas acepciones diferentes recogidas por J. Reis en su clásica monografía, Was ist ein Satz?, Praga, 1931.

3 Para el problema terminológico de estos dos últimos términos, cf. recientemente la documentación aportada por B. Spillner (1992: 607-624) y P. Wunderli (1992: 693-718), respectivamente. 
(como generar o estructura profunda en la gramática transformacional), advierte que ello no siempre constituye una ventaja, pues «puede inducir a error si a los términos se atribuyen el valor y las connotaciones que las mismas formas tienen en las lenguas» (op. cit.: 125). Estos mismos escollos han sido criticados también por otros autores, como J. Fernández Sevilla (1983: 47, 50-51 y $55 \mathrm{n}$. 17), quien destaca cómo, por desgracia, los hechos polisémicos «son frecuentes en un ámbito como el de la terminología científica y técnica, en donde no cabría esperarlas: puede comprobarse la ambigüedad de términos como lexema, lexía, sintagma, etc., cuyo contenido difiere sensiblemente según el autor o la escuela que los emplean». Y respecto a la sinonimia, tras objetar los «razonamientos no del todo convincentes» de K. Baldinger en su negación de la sinonimia terminológica, expresa que ésta, muy limitada tanto en la lengua común como en la lengua literaria, abunda sorprendentemente en la lengua científica, donde puede ocasionar confusiones intolerables (así, entre agudo / llano / esdrújulo y los correspondientes oxitono / paroxitono / proparoxitono, no existen ciertamente diferencias denotativas aunque sí de connotación). Esta falta de univocidad de la terminología lingüística ha sido puesta igualmente de manifiesto por G. Salvador (1985: 126-132, esp. 127-129), que ofrece una abundante muestra de la evidente polisemia y sinonimia terminológicas que caracteriza la lingüística general, lo que suele producir «tremendas confusiones $y$, con frecuencia, notables errores conceptuales», dado que «al lado de términos específicos, creados exprofeso, abundan los habilitados como tales pero con uso libre en la lengua común» (op. cit.: 127).

Por lo que respecta a la semántica, que debería sentar ejemplo de consistencia verbal, podemos paradójicamente advertir que incluso tales dificultades de la proliferación terminológica se agravan aún más, hecho éste que deriva justamente de una falta de precisión, homogeneidad y unificación de criterios en sus orientaciones y principios metodológicos, así como de delimitación y clara definición de su objeto y unidades constitutivas (cf. R. Trujillo 1972: 103 y 1983a: 185 y 212). En esta misma línea, A.-J. Greimas (1966: 6-7) había ya expresado que «la difficulté de déterminer les méthodes propres à la sémantique et de définir les unités constitutives de son objet est réelle (...). La définition traditionnelle de son objet, considéré pudiquement comme «substance psychique», empêchait de la délimiter nettement par rapport à la psychologie et, plus tard, la sociologie». Y en cuanto a sus unidades, «le fourmillement terminologique de sémèmes, sémièmes, sémantèmes, etc. — ne révèle que l'embarras et la confusion. Le linguiste le mieux intentionné ne pouvait, dans ces conditions, considérer la sémantique que comme une discipline qui se cherche».

1. En lo referente al panorama sinonímico, son fácilmente demostrables casos en este sentido dentro del problemático terreno de la nomenclatura lingüística, donde abundan inútilmente y se prestan obviamente a confusiones, 
como sucede con llana y grave dentro de la prosodia del español o con las denominaciones de las funciones del lenguaje o de la palabra como unidad lingüística ${ }^{4}$. Pero, junto a estos ejemplos, comprobamos, de la misma forma, que lo habitualmente señalado para la lengua común respecto a la inclusión en los diccionarios de sinónimos de vocablos que son otra cosa y a la tradición lexicográfica perpetuada hasta nosotros, que consideraba la sinonimia como una relación que consistía en precisar léxicamente los llamados sinónimos estableciendo en la medida de lo posible sus diferencias de significación, puede aplicarse asimismo al lenguaje científico. De este modo, monema y morfema, aparte de las implicaciones sintomáticas alusivas a escuelas lingüísticas diferentes ${ }^{5}$, no son, en principio, términos sinónimos, sino que mantienen, tal como ha expresado L. Wolf (1974: 58), una relación de inclusión. Así, refiriéndose a la idea de E. Coseriu (seguida igualmente por $\mathrm{H}$. Geckeler) de que el vocabulario técnicocientífico está organizado sobre distinciones lógicas basadas en oposiciones exclusivas, este autor señala que «die letzte Behauptung ist äußerst problematisch, da sich in Fachterminologien in der Regel auch beliebig viele inklusive Oppositionen nachweisen lassen, so z.B. in der sprachwissenschaftlichen Terminologie»:

\section{Monem}

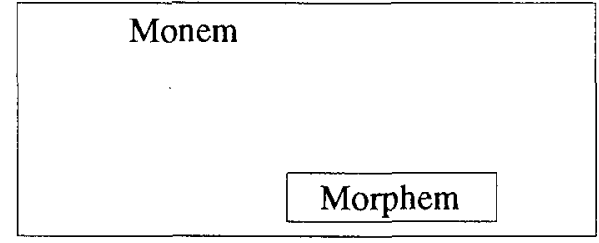

ejemplo, éste, que responde al uso terminológico más extendido dentro de la lingüística europea de orientación francesa desde que A. Martinet (1967: 15-16) distinguiera, en su concepción de las unidades lingüísticas básicas, como tipos de monema, tanto el morfema como el lexema, relación inclusiva que ha sido recientemente representada por $P$. Wunderli (1992: 693) en el siguiente esquema:

4 Para tales sinónimos terminológicos, véase G. Salvador (1985: 59 y 127-129), que desafía a quien le señale la menor diferencia tanto significativa como expresiva entre llana y grave y donde explica, entre otros conceptos lingüísticos, las confusiones teminológicas originadas por la disparidad sinonímica que recubre tanto a las funciones del lenguaje como a la palabra en la terminología lingüística. En cuanto al primer ejemplo, E. de Bustos Tovar apunta, en sus anotaciones a la semántica francesa de S. Ullmann (1974: $284 \mathrm{n}$. 47), que «la polisemia del esp. grave, una de cuyas acepciones indica la acentuación de la palabra en la penúltima silaba, estará complicada con la sinonimia respecto a palabra llana, palabra barítona, término este último empleado por el gramático Salvador Fernández Ramírez».

$5 \quad$ «(..) souvent, les implications symptomatiques sont absolument nécessaires pour garantir la compréhension (monème = école de Martinet, morphème $=$ école de Bloomfield)» (K. Baldinger 1968: 54; cf. también 1977: 234 y 1984: 195). Para la distinción terminológica de estos dos conceptos según sus ámbitos de empleo lingüístico, véase P. Wunderli (1992: 693). 
bedeutungstragende Minimaleinheit

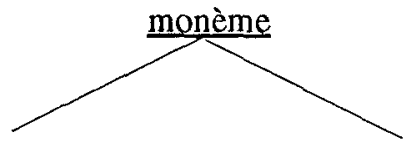

Morphosyntax

Lexikon

morphème

$\underline{\text { lexème }}$

De cualquier forma, el tipo de relación entre estas unidades de la semántica dependerá, en última instancia, del plano de metalengua de cada propio investigador, de su explícita definición metalingüística, con lo que no se excluye, naturalmente, la posibilidad de una caracterización exacta de ambos términos ${ }^{6}$.

Otro caso de coincidencia terminológica que no se corresponde exactamente con una identidad de conceptos es el de semántica y semasiología, que no son actualmente sinónimos, han repartido su significado, aunque sí es cierto que hubo un tiempo (concretamente desde finales del siglo pasado hasta muy entrados los años cincuenta) en que ambos términos se confundieron. Basta un breve repaso a las fuentes doctrinales de esta época para observar, por ejemplo, cómo G. Stern (1931), consciente de la ambigüedad, generalidad y aplicaciones adicionales del término semántica, intentó dejar éste para los estudios extralinguísticos del significado y semasiología para su campo de estudio, pero su lógica propuesta no encontró el apoyo y la resonancia suficiente por el peso específico que el término semántica tenía en Francia, frente al de semasiología. Por su parte, P. Guiraud (1955: 108-109) insiste en este problema al tratar las distintas divisiones del plano de la semántica. Desde su perspectiva, la semasiología (estudio de las significaciones de las palabras desde su forma significante) y la onomasiología (estudio de las palabras partiendo de la cosa significada) constituyen, más que dos partes autónomas de la semántica bajo sus diversas formas, dos puntos de vista distintos. En esta línea señala la necesidad de fijar esta terminología fluctuante y clarificar, especialmente, si la palabra semántica debe abarcar el conjunto de estos problemas o ser reservada para uno de ellos. Incluso el propio S. Ullmann - a quien posiblemente debemos en gran parte el triunfo definitivo de la palabra semántica por su preferencia por esta denominación en sus célebres estudios de la década de los cincuenta sobre el tema (cf. la opinión de H. Geckeler 1983: 52) - llega a afirmar en algunos de sus tratados (cf. 1957: 5 y 1964a: 142) que ambos términos siguen compitiendo todavía y son sinónimos algo engorrosos cuando se aplican a la filología?.

6 «Il faut cependant ajouter que des relations d'inclusion telles que monème $\supset$ morphème, auxquelles se réfère Lothar Wolf (...) n'excluent naturellement pas des définitions exactes» (K. Baldinger 1984: 195 n. 46; también 1977: 234 n. 40). 
Pero esta competencia terminológica no puede ser sostenida hoy día, dado que, con excepción de los planteamientos de ciertos autores ${ }^{8}$ que han continuado esta tradición utilizando semasiología en su significado general, el término semántica va a suplantar, con el paso del tiempo, a este último, que acaba prácticamente por restringir su valor inicial en un sentido paralelamente opuesto al de onomasiología y ampliando aquél no sólo nuevos contenidos desde una visión estrictamente lingüística, sino abarcando toda una extensa gama de problemas de diversa índole que exceden los límites puramente lingüísticos. De esta forma, tanto el término semántica como el que designa su objeto de estudio, el significado, se convierten, pues, en vocablos ambiguos con todo un campo de dispersión significativa que comprende un extenso abanico de especializaciones, matices interpretativos y acepciones terminológicas. Semántica es, por tanto, término inclusivo que comprende tanto la onomasiología como la semasiología, disciplinas específicas 0 , más bien, métodos de análisis con base epistemológica diferente que designan diversas perspectivas, opuestas por la naturaleza de sus correlaciones pero con planteamientos complementarios entre sí, dentro de la semántica?.

Sin embargo, K. Baldinger, que encuentra en la terminología científica las

7 Para una historia de ambos términos en el marco del nacimiento de esta disciplina como descripción científica autónoma, cf. M. Bréal (1883: 133 y 1924: 8); A. W. Read (1948: 78-97, esp. 82); P. Guiraud (1955: 108-109); K. Baldinger (1956: 148-149 y 1957: 4-7); S. Ullmann (1957: 5 y 1964a: 142); E. Coseriu / H. Geckeler (1974: 104-106 y 1981: 8-10); H. Geckeler (1973: 5-6 y 1983: 51-52); M. Martínez Hemández (1984: 356-357); M. Casas Gómez (1991: 114-115 y 120-122, nn. 8 a 18); M. Casas Gómez / Ma D. Muñoz Núñez (1992: 134-135 n. 4), y las referencias citadas en la n. 8.

* Sobre todo de Alemania y de Europa oriental. Véanse al respecto las obras de E. Struck (1940) y (1954); H. Kronasser (1952) y (1968); V. A. Zvegincev (1957); T. Schippan (1972) y (1975), y, de forma especial, la semasiología de K. Baldinger que, si bien es continuadora de la vieja tradición germánica de este término como teoría de la significación (cf. M. Casas Gómez 1991: 114115), puede servimos en sus distintas ediciones (1956: 148-155 y 173-179; 1957, y 1964a) de eslabón de este cambio terminológico que revela una distinta concepción de la disciplina. En este sentido, ya en su versión original (1956: 152 n. 22) apunta que «"Semasiologie" oder "Semantik" hat zwei Bedeutungen, eine umfassendere (Wissenschaft von den Wortinhalten im Sinne Ullmanns) und eine speziellere (Inhaltentwicklung eines einzelnen Wortes)», nota que será completada más tarde en la versión española (1964a: 13 n. 22) de este trabajo ("Aquí se alude al sentido más especializado, pero en el título de este cuadro de conjunto se hace referencia al significado más general (...). Una parte de la controversia sobre la Semasiología puede reducirse al doble aspecto de esta denominación»), que incluye además un apéndice en el que expresa abiertamente la sustitución terminológica de semasiología por semántica: «En el caso de una nueva redacción de este cuadro de conjunto, propondría una separación terminológica nítida entre Semántica y Semasiología, ya iniciada cn la práctica, y que aquí todavía no se ha llevado a cabo (véase la nota 22): Semántica para el sentido más general de Teoría del contenido de la palabra, Semasiología para el sentido más restringido de Teoría de la significación en relación con el cuerpo de la palabra. Por consiguiente el título de este cuadro de conjunto debería cambiarse en La Semántica» (op. cit.: 52).

9 Como ha advertido H. Geckeler (1983:52), «en la terminología usual de hoy, «semasiología» se entiende sobre todo como término opuesto a «onomasiología» (...), de modo que no hay que confundir «semasiología en sentido amplio» (equivalente a «semántica») con "semasiología en sentido estricto» (opuesta a «onomasiología»)». Para un examen de las relaciones entre onomasiología y semasiología como «deux méthodes de recherche linguistique, plutôt que deux disciplines», véase el trabajo de P. Swiggers (1983: 431-438). 
mayores dificultades para defender su punto de vista ${ }^{10}$ sobre la existencia de sinónimos absolutos sólo en el plano simbólico-conceptual u onomasiológico y nunca en el semasiológico (donde interviene toda una serie de factores diferenciadores de carácter sintomático y signalético), llega a no admitir la sinonimia en el campo terminológico desde este último plano, pues, aun cuando en tales casos el contenido conceptual se sitúa en primera línea, ello no impide el que siempre aparezcan valoraciones estilísticas, aunque implícitamente estén relegadas. De esta forma, ejemplificando con terminología lingüística, destaca dos factores entre los estudiados por él que rebasan la estructura del monema, como son la semejanza o parentesco formal de uno de ellos con otros monemas en el plano de la expresión y la motivación morfológica de los sinónimos. Bajo este prisma, semántica y semasiología, empleados como sinónimos, se distinguen desde el punto de vista estilístico por el hecho de que este último está formalmente emparentado con onomasiología ${ }^{11}$, y Bedeutungslehre frente a Semantik o Semasiologie se diferencian en que el primero es motivado - como palabra compuesta de la lengua alemana - y los otros son préstamos extranjeros ${ }^{12}$. A este respecto, no le falta razón a S. Ullmann (1964a: 142) cuando advierte que entre tales sinónimos científicos apenas se puede hablar de diferencias estilísticas ${ }^{13}$, puesto que se usan en los mismos contextos e incluso hasta en los títulos de un

to Cf. K. Baldinger (1968: 53-54; 1977: 234, y 1984: 194-195), cuyo planteamiento es coincidente con el de L. Söll (1966: 94), quien, tras citar algunos de los ejemplos aquí analizados, concluye que «die Synonymie der Fachsprachen ist also eine Synonymie sui generis und als solche äußerst labil».

11 Cf. K. Baldinger (1968: 53 n. 33; 1977: 234 n. 39, y 1984: 194 n. 45) y también Y. Muñoz Valle (1975) que, en repetidas ocasiones (p. 265 n. 3 ; p. 271 n. $13 ;$ p. 280 n. 22, y p. 282 n. 29), comenta este ejemplo en sus notas críticas a la concepción sinonímica sustentada por el lingüista suizo.

12 Lo mismo ocurre con Lautlehre y Phonetik o Formenlehre y Morphologie (cf. L. Söll 1966: 94: indica que «Experimentelle Laullehre für experimentelle Phonetik ist nicht möglich»; K. Baldinger 1968: 54; 1977: 234, y 1984: 195, e I. Muñoz Valle 1975: 265 n. 3, 271-272 n. 13, 280 y 282 n. 29).

13 Este autor, partiendo del hecho de que las nomenclaturas técnicas y científicas están claramente definidas por rasgos objetivos pertenecientes a los «objetos reales» y poseen una precisa delimitación de acuerdo con la realidad que designan, opina sin reservas que en este sector del vocabulario se pueden encontrar abundantes ejemplos de términos sinónimos completamente intercambiables (cf. S. Ullmann 1952: 180; 1957: 109; 1964a: 141-142, y 1964b: 74). En esta línea, suministra ejemplos como semántica - semasiologia (en la teoría de la significación), cecitis - tiflitis (en medicina; cf. también C. Schick 1960:188) y espirante - fricativa (en fonética). Para este último doblete, véase J. M. Blecua (1973: 68), que alude al caso de articulación fricativa como tecnicismo sinónimo de espirante (cf., en cambio, O. Duchácek 1979: 18-19, quien, siguiendo a A. Martinet, aprovecha esta molesta e inútil sinonimia para precisar nociones semejantes, distinguiendo semánticamente los términos consonne constrictive, consonne fricative y consonne spirante). Esto no quiere decir en modo alguno que el lingüista checo no proporcione sinónimos perfectos absolutos - de acuerdo con su tipología sinonímica (1964: 37 y 1967:55-56) - en la terminología científica, donde opina que son bastante frecuentes. Así, por ejemplo, no existe a su juicio diferencia entre voyelles vélaires y voyelles postérieures o entre voyelles mixtes, voyelles labialisées y voyelles arrondies. Por otra parte, abundantes casos análogos, algunos más bien variantes morfológicas que sinónimos propiamente dichos, así como la explicación de determinadas fuentes generadoras de la sinonimia en la terminología, son señalados por este autor en el trabajo anteriormente citado (1979: 9-19). 
mismo libro, como ocurre con los manuales clásicos de H. Kronasser, Handbuch der Semasiologie. Kurze Einführung in die Geschichte, Problematik und Terminologie der Bedeutungslehre (1952, 2 ed. 1968) o de E. Struck, Bedeutungslehre. Grundzüge einer lateinischen und griechischen Semasiologie (1940, 2a ed. 1954), claros exponentes de la tradición historicista del modelo germánico de teoría de la significación inaugurado hacia 1825 - en el seno de la filología latina - por C. K. Reisig (1839: 286-307) con su estudio «Semasiologie oder Bedeutungslehre» ${ }^{14}$. Por otra parte, en lo que se refiere a la existencia en Alemania del término compuesto Bedeutungslehre, comenta $\mathrm{H}$. Geckeler (1983: 51 n. 3) que éste «no tiene equivalente ni en las lenguas románicas ni en inglés y que significa literalmente «ciencia del significado»», lo cual coincide con la definición de sémantique propuesta por M. Bréal, «que, en realidad, es nada más que una traducción del término alemán». Desde nuestro punto de vista (cf. también M. Casas Gómez 1991: 114, 120-121 n. 8 y 122 n. 16), más que una traducción del vocablo germánico, su concepción de la semántica como «science des significations» y de «les lois qui président à la transformation des sens» (M. Bréal 1883: 133 y 1924: 8) no es sino una definición paralela que traduce prácticamente el concepto de semasiología (ciencia de la investigación del significado y de los «Grundsätze, welche bei der Entwicklung der Bedeutung gelten») desarrollado años antes por C. K. Reisig (1839: 286; cf. L. Antal 1972: 21). Además, no deja de ser significativo que el lingüista suizo, en este apartado que dedica a la sinonimia en la terminología científica, se limite a establecer diferencias estilísticas entre ambos términos, cuando es él justamente uno de los principales autores que han contribuido a la consolidación práctica de tales diferenciaciones terminológicas. Aparte de las ideas contenidas en su Semasiología (cf. n. 8), en su artículo específico sobre los métodos semasiológico y onomasiológico (incluido como apéndice en su Teoría semántica), habla conscientemente, a propósito de la clarificación terminológica sugerida por P. Guiraud, de la doble significación - ahora ya anticuada - de la palabra semasiología, al distinguir entre semasiología, disciplina que considera el desarrollo de las significaciones de las palabras aisladas (campo semasiológico), onomasiología, disciplina que «envisage les désignations d'un concept particulier, c'est-à-dire, une multiplicité d'expressions formant un ensemble» (campo onomasiológico) y semántica, a la que define como ciencia del contenido del lenguaje, sentido más amplio que abarca tanto la semasiologia como la onomasiología (cf. K. Baldinger 1964b: 250 n. 1 y 269 n. 1 y 1977: 268 n. 3 y 294 n. 4). Tales distinciones han sido transmitidas asimismo por su discípulo K. Heger, que los separa perfectamente desde un punto de vista

14 Trabajo que aparece recogido también en el libro de L. Antal (1972: 19-40). Para algunos datos acerca del nacimiento de esta ciencia y de los seguidores de este filólogo clásico, véanse las referencias citadas en las nn. 7 y 8 . 
semántico, advirtiendo que «los dos términos semántica y semasiología no son sinónimos sino para quien por motivos de principio cree que debe poner en duda la legitimidad de la onomasiología» (1974: 1 n. 1). Previamente, en esa misma nota, había subrayado su diferenciación en lo relativo a la terminología utilizada:

«De acuerdo con una costumbre cada vez más arraigada, hago conscientemente la distinción entre semántica y semasiología. Como término genérico, semántica comprende todos los dominios de la lingüística que de algún modo u otro tienen por objeto la función significativa de los monemas (...). Dentro de esta Semántica y, por tanto, como término específico, la semasiología se define por la oposición que forma con la onomasiología. Comprende todos los dominios en los que el punto de partida es el monema o un grupo de monemas y en donde el objeto del estudio es la significación, el significado, etc., de este monema o monemas» ${ }^{15}$.

En el marco de la historiografía de estos términos puede observarse que, en lo que atañe a sus definiciones y evolución de sus contenidos, los estudios de semasiología o semántica, desde sus orígenes y, más concretamente, desde finales del siglo pasado en el que adquirieron carácter científico, sobre todo a raíz del auge y desarrollo que éstos tuvieron en el transcurso de la década de los sesenta, se han limitado prácticamente, al menos en la lingüística europea, al nivel propiamente léxico, existiendo incluso, entre los funcionalistas, algún autor que ha precisado no hace mucho que «la semántica no es la ciencia de los significados en general» sino en realidad «la ciencia de los significados léxicos» (H. Geckeler 1983: 51), concepción que en el estado actual de las investigaciones en este campo resulta plenamente inaceptable, por cuanto la lexicología (semántica léxica) constituye tan sólo una parte de la semántica lingüística, que abarca genéricamente el estudio global del plano del contenido y debe encargarse de estudiar el significado en un nivel inferior al estrictamente léxico y en otros superiores a éste, con lo que podemos hablar de una semántica morfológica o gramatical, de una semántica léxica o lexicología, de una semántica del grupo de palabras, de una semántica sintáctica u oracional y de una semántica textual; en definitiva, de un análisis del significado según los distintos niveles lingüísticos.

2. De esto se infiere que nuestro metalenguaje no se compone de signos unívocos. Todo lo contrario. En la lingüística, los términos técnicos experimentan una especialización significativa — bien individual (según el estudioso que los defina) o bien por escuela - de su contenido objetivo, siendo abundantes las expresiones textuales ambiguas y las acepciones polisémicas de carácter terminológico. Más que la sinonimia o la hiperonimia-hiponimia, de las que ya

is Para el desarrollo de su concepción tanto de la semasiología como de la onomasiología, véanse las tesis expuestas en sus respectivos modelos trapezoidales (1965: 7-32; 1969a: 47-111; 1969b: 44$66 ; 1971: 22-48 ; 1974: 1-32$ y $136-207$, y $1981: 61-92$, esp. 86-89). 
hemos consignado algunos ejemplos, será la polisemia ${ }^{16}$ la que caracterice primordialmente la terminología de esta ciencia de la cultura. Un caso extremo de lo que venimos diciendo lo constituye el término significación, que ha llegado a ser un tecnicismo sumamente polisémico, tal vez el más polisémico de la nomenclatura lingüística. Ya S. Ullmann (1952: 210 y 1964a: 172) comentaba que la ambigüedad de este término ha supuesto tanto una rémora para el progreso de la semántica que algunos investigadores han preferido evitarlo por completo $^{17}$. No obstante, dos son los sentidos básicos con que se utiliza el término significación: en cuanto correlato del significante o en cuanto relación (cf. S. Gutiérrez Ordóñez 1981: 116). Así, para K. Baldinger (1964b: 253 y ss., esp. $268-271$; 1977: 39-40, 119-121 y 273-294, y 1984: 12-13, 90-92 y 228247), significación se identifica fundamentalmente ${ }^{18}$ con campo semasiológico (al que corresponde inversamente el campo onomasiológico o de designaciones), con lo que los conceptos de significación y designación desarrollados por este autor no son en modo alguno equivalentes - como aclara explícitamente el lingüista suizo ${ }^{19}$ - a los utilizados por E. Coseriu, o, si seguimos los planteamientos de S. Ullmann (1952: 22-23; 1956: 12-20, esp. 14; 1957: 69-70; 1964a: 57; 1964b: 18; 1973: 6-7, y 1974: 28-29) en su remodelación teórica del triángulo metodológico de C. K. Ogden e I. A. Richards (1960, cap. I: 10-11), observamos cómo este autor denomina significación a la relación reversible y de evocación recíproca que manifiestan los dos componentes del signo lingüístico: significante y significado, de acuerdo con la terminología saussureana. De esta

16 Para K. Baldinger (1977: 59 y 1984: 30-31), que no ve límites muy claros en las diferenciaciones coserianas entre un léxico estructurado y un léxico nomenclator y teminológico, «en realidad, el lenguaje científico se sirve del lenguaje común y muchas veces no puede esquivar sus implicaciones de polisemia, homonimia, etc. Muchos debates de terminología son la consecuencia de este hecho».

17 Consúltense, por ejemplo, las numerosas acepciones recogidas por C. K. Ogden e I. A. Richards (1960, cap. IX: 186 y ss.) y W. P. Alston (1974: 25-27). Un resumen de las teorías sobre la significación, así como un análisis de los distintos estratos del significar, puede verse en $S$. Gutiérrez Ordóñez (1981: 107-133 y 1989: 57-70).

18 Ya que el término se corresponde, en ocasiones, con el concepto de significación desarrollado por S. Ullmann y, a veces, es equivalente también al significado saussureano. A propósito de estas dos equivalencias, $M^{a}$ D. Muñoz Núñez (1993: 117 n. 12) advierte cómo tales correspondencias «quedan invalidadas al identificar $\mathrm{K}$. Baldinger la significación, por una parte, con el «significado», «semerna», «concepto» u «objeto mental» individual ligado a una forma, y, por otra, con la relación ya mencionada que va de un significante a un concepto o a un conjunto de ellos (lo que supone, al mismo tiempo, la identificación de significación con campo semasiológico), relación que, por tanto, se da en una única dirección».

19 En dos notas de su Teoría semár.ica (1977: $57-58$ n. 18 y 119 n. 1 y $1984: 29$ n. 23 y 90 n. 1), este autor hace constar que, desde su especulación teórica, el empleo de significación y designación no coincide con la terminología coseriana, si bien las equivalencias que establece suponen una distinta interpretación de la concepción expresada por el lingüista rumano: «se ve aquí que Coseriu se sirve de los términos significación (= designatio, se refiere a conceptos intensionalmente definidos) y designación (= denolatio, se refiere a conceptos extensionalmente definidos) de otra manera que nosotros (...) nuestra oposición significación / designación queda dentro del 'designatum' y no corresponde a la oposición designatum / denotatum». 
forma, se advierte que el uso de este término no se corresponde con el empleado por el maestro ginebrino (1922: 99 y 158-162, esp. 158), para quien es equivalente de significado, esto es, la contraparte de la imagen auditiva o significante $^{20}$. Su utilización desde aquella perspectiva ofrece la ventaja de superar, ya desde una concepción de la semántica tradicional o preestructural, la posible sinonimia terminológica entre significado y significación. Más tarde, dentro de una óptica estructural-funcional, E. Coseriu ${ }^{21}$ limitará, en cambio, esta relación al plano semántico y no al plano de correlación significado-significante, distinguiendo entre significado, en cuanto nivel del significar junto a la designación y al sentido, y significación, como relación estructural entre significados de signos opuesta a la relación de designación entre los signos lingüísticos y los objetos ${ }^{22}$. Por estos y otros rasgos diferenciales, significación, significado y sentido, que pueden usarse indistintamente en el lenguaje corriente como términos equivalentes, no deben serlo de ningún modo para un semantista, pese a que se hayan empleado incluso metalingüísticamente en distintas lenguas (Bedeutung / Sinn, signification / signifié I sens, meaning / sense, etc.), como «sinónimos» en la tradición de los estudios semánticos, sobre todo por parte de la semántica histórica y preestructural, así como por muchos de los autores encuadrados dentro de una óptica estructural que no comparten la visión ortodoxa del estructuralismo en sentido estricto ${ }^{23}$. De hecho, la distinción entre significado y sentido se convierte concretamente en

20 Véase, de este modo, la distinción que este autor propone entre significación y valor, términos que en modo alguno debemos utilizar como sinónimos (cf. R. Godel 1957: 275-276 y 280-281 y R. Engler 1968: 45-46 y 52-53). En relación con esta diferencia saussureana, que separaba nítidamente el concepto o representación de una «idea» de la estructura significativa interna, comenta acertadamente R. Trujillo (1988: 123): «A la estructura conceptual, Saussure opone la propiamente idiomática: la significación (es decir, lo que yo llamo estructura conceptual) está bajo la «dependencia» del valor (lo que yo llamo estructura semántica)».

21 Cf. 1964: 145-146, en las que parte de la noción de significación expuesta por S. Ullmann como relación entre la expresión y el contenido para establecer algunas consideraciones sobre el cambio semántico; 1966: 208-210; 1967: 13-17 y 21-22 n. 4; 1968:3; 1969: 55-56; 1970: 104-121; 1973: 49-50; 1976: 5-7; 1977a: 21-22, 130-133, 162-163 y $185-209 ; 1977 \mathrm{~b}: 25-29,39-45,53-54 \mathrm{n}$. 4 (donde explica el uso, como términos técnicos, de la distinción tripartita entre significado, designación y sentido, para referirse a tres niveles de funcionalidad o tipos diferentes de contenido), 219-222 (con aplicación a la teoría de la traducción), 243-249 y 258; 1978: 25, 117-118, 129, 135-143, 187 203 y 206-209; 1980: 43-50; 1981a: 283-286, y 1981b: 13-14. En colaboración con H. Geckeler, véanse asimismo sus estudios (1974: 146-147 y 1981: 54-55); también los trabajos de este último (1971: 78-83 y $1973: 2-3$ y 16$)$.

22 Para los sentidos diferentes que estos téminos y relaciones adquieren en la semántica «tradicional» o preestructural en oposición a lo que tales conceptos indican dentro del estructu ralismo y funcionalismo semánticos, así como para un análisis de las posibilidades de implicación léxica de estos niveles del significar, véase nuestra contribución al homenaje del Prof. Horst Geckeler (1994a, en prensa).

23 Para algunos datos acerca de esta identificación, cf. M. Casas Gómez / Ma D. Muñoz Núñez (1992: 135 n. 7) y M ${ }^{2}$ D. Muñoz Núñez (1993: 57 n. 3; 116-118 nn. 9 y 12, y 209-210 n. 41). En concreto, la confusión entre signification y sens en el metalenguaje de la lengua francesa ha motivado la introducción de nociones particulares, como la de signifiance (significación en la lengua) y sens (significación en el discurso), distinción propuesta por E. Benveniste (cf. T. Todorov 1975: 509. 515). 
uno de los ejes centrales que motiva la discusión en torno al problema sinonímico.

3. Con el análisis de estos términos lingüísticos, cuyos materiales forman parte de un futuro diccionario de terminología semántica que actualmente proyectamos, hemos pretendido abordar cómo se presentan determinadas relaciones léxicas en este ámbito terminológico, con objeto de poder reflexionar sobre las características semánticas propias de este léxico técnico en relación con los principios que rigen otras terminologías científicas.

En lo que al primer punto se refiere, ya en distintos trabajos en los que revisamos teóricamente los principios lexemáticos que caracterizan a los términos de las ciencias y las técnicas (cf. M. Casas Gómez 1993 y 1994b), hemos indicado la existencia de sinónimos totales o perfectos en este tipo de vocabulario, opinión sobre la que existe en líneas generales, contrariamente a lo que ocurre en el léxico común, unanimidad de criterios en la lingüística actual. Según hemos podido comprobar por los ejemplos aducidos, la sinonimia en este dominio - si existe como tal entre dos o más términos - siempre es absoluta, al margen de las posibles diferencias estilísticas o evocativas que continuamente puedan darse entre ellos, implicaciones que no deben ser consideradas desde una visión estrictamente semántica. Ahora bien, insistimos en que debe existir realmente una auténtica relación sinonímica, pues en ocasiones apreciamos casos de no coincidencia semántica en el léxico nomenclator, tal como hemos intentado demostrar que ocurre a menudo en la terminología linguística, hecho éste motivado principalmente por dos razones: 1) porque a veces se suelen citar como sinónimos términos que en el metalenguaje de nuestra ciencia no lo son en modo alguno, de forma que se hacen coincidir terminológicamente vocablos técnicos que no representan una identidad conceptual ${ }^{24}$, y 2) porque algunos autores intentan diferenciar, sobre todo estilísticamente, parejas de «pretendidos» sinónimos terminológicos (precisamente con el propósito de negar la sinonimia en el ámbito científico y técnico), cuyos valores objetivos no se corresponden exactamente.

En este sentido, conviene que nos detengamos por un momento a reflexionar sobre las características terminológicas propias de la ciencia lingüística. Como consideración de carácter general para el ámbito técnico-científico, E. Coseriu (1966: 182; también 1987: 177-178) sostiene que las oposiciones terminológicas son exclusivas de acuerdo con el principio de [no-]contradicción, según el cual es inconcebible «qu'un terme soit le contraire d'un autre, et, en même temps, englobe son contraire». Este rasgo distinguiría el lenguaje técnico del lenguaje

24 A esta misma conclusión 1lega G. Salvador (1985: 127) en su búsqueda de discrepancias terminológicas y revisión de la terminología de lingüística general de los manuales de lengua española del bachillerato: «Las encontramos, qué duda cabe, pero lo que vimos en seguida es que esas discrepancias en los términos comportan frecuentemente ambigüedades conceptuales y que las coincidencias terminológicas no siempre representan identidad de conceptos». 
propiamente dicho donde las oposiciones - según el lingüista rumano - son con mucha frecuencia inclusivas, distinción que - como le ha sugerido certeramente R. Trujillo (1974: 207-208) — «no llega a establecer formalmente la frontera de una manera precisa», pues se trata sólo de un rasgo que caracteriza lingüísticamente a muchas oposiciones léxicas, pero no a todas, ya que existen oposiciones exclusivas, «rigurosamente lingüísticas», en el léxico «estructurado». Tal circunstancia obliga al lingüista español a señalar otras características formales propias de este léxico, como las implicaciones paradigmáticas y sintagmáticas que contraen las palabras en oposición a los términos del léxico «ordenado».

Sin embargo, hemos hablado de relaciones inclusivas en la ciencia del lenguaje, lo que parcialmente vendría a modificar el mencionado postulado de la no-contradicción en tanto exigencia propia de toda terminología científica, al menos en lo concerniente a determinados dominios terminológicos en los que la hiperonimia jerarquiza toda una serie superordenada de sucesivas relaciones de inclusión entre ciertas unidades léxicas de carácter técnico o científico. En este sentido, en sendos trabajos publicados en un número relativamente reciente de Langages (editado por M.-F. Mortureux) sobre el tema monográfico «L'hyponymie et l'hyperonymie», P. Lerat (1990: 79-86) y D. Jacobi (1990: 103-114) han destacado la importancia de la hiperonimia - cohiponimia - hiponimia, junto con otras relaciones lógicas y semánticas, en la «estructura» general de las terminologías y en el discurso de vulgarización científica, con ejemplos de dominios tan distintos como la «explotación forestal» (cuyo esquema de análisis lo toma P. Lerat de P. Auger / L.-J. Rousseau 1977: 18) y la «inmunología celular», respectivamente ${ }^{25}$. De hecho, ya L. Wolf (1974: 58) había insistido en que la ausencia de oposiciones inclusivas o la presencia única de oposiciones exclusivas no podía ser considerada como característica de las terminologías especiales, con lo que había que evitar una identificación de éstas con las llamadas nomenclaturas enumerativas, basadas en diferenciaciones exclusivas. Según esta constatación, concluía, en la línea expuesta anteriormente, con la siguiente hipótesis:

«Wenn hier ein Unterschied zum fachlich nicht fixierten Wortschatz bestehen soll, so ist er sicher nicht qualitativer, sondern bestenfalls quantitativer Art, da in beiden unterschiedenen Teilen des Wortschatzes jeweils inklusive und exklusive Oppositionen gleichermaßen vorkommen. Aber auch ein nur quantitativer Unterschied bliebe erst noch nachzuweisen».

25 En la misma línea, S. Jacobson (1984: 355-361), que intenta mostrar cómo los límites establecidos por E. Coseriu entre las terminologías y el lenguaje usual no existen en realidad, cita un ejemplo más de relación inclusiva, esta vez procedente del campo de la energía eléctrica: «electron as a superordinate unmarked term covering both the marked term electron ${ }_{2}$, with its synonym negatron, and the term positrons (op. cit.: 357 ). 
Por otra parte, sabemos que uno de los principios lexemáticos propios del lenguaje técnico consiste en que sus términos son meros representantes o sustitutos de las cosas (la significación coincide con la designación), en oposición a las palabras del lenguaje común, en donde ambos niveles —bien como relaciones del significar o en cuanto estratos diferentes de contenido- se presentan por separado. En este sentido, los significados de los términos científicos, frente a los de los signos estructurados o estructurables en la lengua que no pueden ser ni definidos, sólo «mostrados» en su red particular y exclusiva de relaciones intralingüísticas (paradigmáticas y sintagmáticas), ni traducibles, son, por el contrario, «definidos» y traducibles: constituyen signos derivativos que responden a una convención verbal previa, de suerte que dependen de una definición explícita que apunta hacia una realidad determinada e inconfundible (cf. E. Coseriu 1966: 182-183; 1977a: 96-98, y R. Trujillo 1974: 206-210; 1983b: 617-623, esp. 621; 1988, cap. XIII: esp. 134-142).

De estos rasgos dependientes del distinto modo de significar del léxico nomenclator respecto al léxico estructurado, nos interesa destacar aquí el problema de la traducibilidad e intraducibilidad de estas dos clases de signos verbales, en concreto el carácter traducible de las terminologías, que pueden ser - como expresamente había afirmado E. Coseriu (1966: 183) - «traduites sans difficulté dans toute communauté qui possède les mêmes sciences et techniques au même degré de développement, puisque «traduction» signifie dans ce cas simplement «remplacement des signifiants», et non pas «transposition des signifiés d'une langue dans les signifiés d'une autre langue»». Esta idea apuntada por el lingüista de Tübingen le lleva a R. Trujillo (1974: 208) a criticar el punto de vista manifestado por S. Gili Gaya (1964: 274), cuando, en relación con la abundante incorporación de tecnicismos exóticos, propone como solución más recomendable la traducción de términos técnicos mediante palabras de la lengua común, propuesta con la que no está, naturalmente, de acuerdo el profesor de La Laguna, dado que los tecnicismos «entran en la categoría de los «definibles», y de ahí su carácter universal: apuntan sólo a una sustancia, idéntica para todas las comunidades lingüísticas (el lenguaje técnico siempre podrá ser traducido a otra lengua sin residuo) (...). Traducir el tecnicismo es, hasta cierto punto, «destecnificarlo» y pasar de un nivel semiológico a otro» (R. Trujillo 1974: 210).

Pero el hecho de que la traducción en el ámbito terminológico no deje residuo semántico alguno está lejos de cumplirse, en cambio, en el campo de la lingüística, debido a los aspectos peculiares que caracterizan semánticamente a sus términos. En efecto, porque muchos son rechazables por innecesarios, ya que suponen la existencia de varios significantes léxicos o usos técnicos para un mismo concepto, y porque añaden confusión terminológica por su falta de precisión y univocidad, la traducción de tecnicismos lingüísticos requiere un 
esfuerzo adicional que conjugue «su necesidad, precisión y adecuación con un cuidadoso proceso de formación del nuevo término ${ }^{26}$. Piénsese, por citar sólo un ejemplo, en el sinnúmero de dificultades a que lleva el traducir meaning por «significación», «significado» o «Bedeutung», sin ulteriores precisiones, como ocurre en las distintas versiones de algunos trabajos de S. Ullmann (cf. 1952: 22-23; 1956: 14, y 1974: 28-29), en los que a una misma definición se les aplica, indistintamente, los conceptos de meaning o signification (significación), y muy a menudo en la lingüística norteamericana, donde meaning «se refiere la mayoría de las veces a lo entendido extralingüísticamente (es decir, a lo «designado»), o se trata de un concepto más general, que abarca designación y significado» (E. Coseriu 1978: 129). Y estas confusiones aumentan desde el momento en que los conceptos «significado» y «sentido», a los que ya nos hemos referido, se identifican por lo general en nuestro metalenguaje. Así, el uso de sense por meaning en la teoría semántica de J. Lyons (1977, I: 174-229; cf. también 1968: 424-428) motiva que R. Cerđà traduzca en ambas obras el término inglés por «sentido», en contextos donde claramente se debería haber empleado meaning ( «significado») o conjunto de diversas relaciones u oposiciones que cada unidad contrae con otras en el sistema. De esta forma, se explica con toda justificación que S. Alcoba recoja, en un artículo específico sobre este tema (1983: 143-152), ciertas reflexiones a propósito de la traducción y adaptación del texto de J. Lyons (1981), en el que argumenta «las soluciones adoptadas en dicha traducción para determinados tecnicismos lingüísticos: en unos casos, porque la solución puede ser alternativa de otra también correcta, en otros, porque difiere de la adoptada en otras traducciones» (op. cit.: 144), con ejemplos, entre otros, como «entrañamiento ${ }^{27}$ para la relación lógica de entailment, traducida por otros autores como «implicación» (cf. W. Abraham 1981: 247-248), «vinculación» (como hace R. Cerdà en sus traducciones de J. Lyons 1977 y R. Kempson 1977) o «entrañe» (véase la versión de J. L. Tato G. Espada de la Semántica de G. Leech 1974), y «comprensión» por «intensión» (intension), contrariamente a la solución adoptada en el texto español de J. Lyons (1977) y en el de W. Abraham (1981: 257), que supone la incorporación de un doblete («comprensión» — «intensión», opuesto a «extensión») de distinta procedencia, como anglicismo del todo innecesario.

Así pues, por lo que respecta a una ciencia de la cultura como la lingüística,

26 S. Alcoba (1983: 151). Para este autor, éstas constituyen «las tres condiciones que ha de satisfacer la introducción de un nuevo tecnicismo en la lengua: necesidad, porque la lengua carezca realmente del término o del término y concepto específico; precisión, porque ha de ser unívoco y distinto, suficientemente diferenciado; y adecuación, porque esté conforme al nuevo concepto o acepción y sea lo más transparente posible respecto al concepto designado».

27 Véase, asimismo, la traducción de F. Meno Blanco del repertorio de terminología língüística actual de W. Abraham (1981: 172-173) e indirectamente también la versión española del diccionario de T. Lewandowski (1982: 184-185, s.v. implicación), al referirse este autor a la distinción propuesta por J. L. Austin - entre implicación, presuposición y entrañamiento (entailment). 
podemos subrayar que, con exclusión de su capacidad sinonímica, común a otros tipos de nomenclaturas, su ambigüedad conceptual, su especialización polisémica (hechos, estos últimos, que suscitan problemas de adaptación en la traducción de sus tecnicismos) y, de forma particular, su peculiar característica de establecer relaciones inclusivas entre sus términos técnicos ponen al descubierto que esta clase de terminologías muy frecuentemente «se trouve à mi-chemin entre le langage général et la terminologie univoque» (K. Baldinger 1968: 54 y 1984: 195), idea ésta que el profesor de Heidelberg defiende con carácter general para todas las terminologías científicas ${ }^{28}$, si bien después los ejemplos que aduce se refieren con exclusividad a la nomenclatura lingüística.

\section{Referencias bibliográficas}

Abraham, W. (1981), Diccionario de terminología lingüística actual. Versión española de F. Meno Blanco, Madrid, Gredos.

Alcoba, S. (1983), «La adaptación de tecnicismos lingüísticos», Cuadernos de Traducción e Interpretación, 3, pp. 143-152.

Alston, W. P. (1974), Filosofía del lenguaje, Madrid, Alianza.

Antal, L. (1972), Aspekte der Semantik. Zu ihrer Theorie und Geschichte 16621970, Frankfurt am Main, Athenäum Verlag.

Auger, P. y Rousseau, L.-J. (1977), Méthodologie de la recherche terminologique, Québec, Régie de la langue française.

Baldinger, K. (1956), «Die Semasiologie. Versuch eines Überblicks», Forschungen und Fortschritte, 30,5, pp. 148-155 y 30,6, pp. 173-179.

Baldinger, K. (1957), Die Semasiologie. Versuch eines Überblicks, Berlin, Akademie-Verlag.

Baldinger, K. (1964a), La semasiología. Ensayo de un cuadro de conjunto, Rosario, Universidad Nacional del Litoral.

Baldinger, K. (1964b), «Semasiologie et onomasiologie», Revue de Linguistique Romane, 28, pp. 249-272.

Baldinger, K. (1968), «La Synonymie, problèmes sémantiques et stylistiques», Elwert, T. (ed.), Probleme der Semantik, Wiesbaden, Franz Steiner Verlag, pp. 41-61.

Baldinger, K. (1977, 2ª ed.), Teoría semántica. Hacia una semántica moderna, Madrid, Alcalá.

Baldinger, K. (1984), Vers une sémantique moderne, Paris, Klincksieck. Blecua, J. M. (1973), Lingüística y significación, Barcelona, Salvat Editores. Bréal, M. (1883), «Les lois intellectuelles du langage. Fragment de sémantique»,

28 En su opinión, «seules les langues artificielles (Algol, etc.) contiennent des unités univoques, à l'opposé (par définition) des terminologies scientifiques qui se servent des langues naturelles» (K. Baldinger 1968: 54; también 1977: 234 y 1984: 195). 
Annuaire de l'Association pour l'encouragement des études grecques en France, Paris, Maisonneuve et Cie, Libraires-Éditeurs, pp. 132-142.

Bréal, M. (1924, $7^{\text {a }}$ ed.), Essai de sémantique. Science des significations, Paris, Librairie Hachette.

Cardona, G. R. (1991), Diccionario de lingüística. Edición española a cargo de $\mathrm{M}^{\mathrm{a}} \mathrm{T}$. Cabello, Barcelona, Ariel.

Casas Gómez, M. (1991), «Panorama actual de la semántica en la filología latina española contemporánea», Excerpta Philologica Antonio Holgado Redondo sacra, I,1, Cádiz, Univ. de Cádiz, pp. 113-153.

Casas Gómez, M. (1993), «En torno a algunos problemas semánticos de la terminología», Translatologica Pragensia, 7, en prensa.

Casas Gómez, M. (1994a), «Implicaciones léxicas de los niveles del significar», Festschrift Horst Geckeler, Münster, en prensa.

Casas Gómez, M. (1994b), «Relaciones y principios lexemáticos en el ámbito de las terminologías», Pragmalingüística, 2, en prensa.

Casas Gómez, M. y Muñoz Núñez, Mª D. (1992), «La polisemia y la homonimia en el marco de las relaciones léxicas», Wotjak, G. (ed.), Estudios de lexicología y metalexicografía del español actual, Tübingen, Max Niemeyer Verlag, pp. 134-158.

Coseriu, E. (1964), «Pour une sémantique diachronique structurale», Travaux de Linguistique et de Littérature, 2,1, pp. 139-187.

Coseriu, E. (1966), «Structure lexicale et enseignement du vocabulaire», Actes du premier Colloque International de Linguistique Appliquée, Nancy, 1964, pp. 175-217.

Coseriu, E. (1967), «Das Phänomen der Sprache und das Daseinsverständnis des heutigen Menschen», Die Pädagogische Provinz, 21,1-2, pp. 11-28.

Coseriu, E. (1968), «Les structures lexématiques», Elwert, T. (ed.), Probleme der Semantik, Wiesbaden, Franz Steiner Verlag, pp. 3-16.

Coseriu, E. (1969), «La struttura del lessico», La Grammatica. La Lessicologia. Atti del I e del II Convegno di studi, Società di Linguistica Italiana, Roma, pp. 55-72.

Coseriu, E. (1970), «Bedeutung und Bezeichnung im Lichte der strukturellen Semantik», Hartmann, P. y Vernay, H. (eds.), Sprachwissenschaft und Übersetzen. Symposion an der Universität Heidelberg (1969), München, Max Hueber Verlag, pp. 104-121.

Coseriu, E. (1973), Probleme der strukturellen Semantik, Tübingen, Gunter Narr Verlag,

Coseriu, E. (1976), «L'étude fonctionnelle du vocabulaire. Précis de lexématique», Cahiers de Lexicologie, 29, pp. 5-23.

Coseriu, E. (1977a), Principios de semántica estructural, Madrid, Gredos. Coseriu, E. (1977b), El hombre y su lenguaje. Estudios de teoría y metodología 
lingüística, Madrid, Gredos.

Coseriu, E. (1978), Gramática, semántica, universales. Estudios de lingülística funcional, Madrid, Gredos.

Coseriu, E. (1980), Textlinguistik. Eine Einführung, Tübingen, Gunter Narr Verlag. Coseriu, E. (1981a), Lecciones de lingüística general, Madrid, Gredos.

Coseriu, E. (1981b), «La socio- y la etnolingüística: sus fundamentos y sus tareas», Anuario de Letras, 19, pp. 5-30.

Coseriu, E. (1987), «Palabras, cosas y términos», In Memoriam Inmaculada Corrales, I. Estudios lingüísticos, 1, La Laguna, Univ. de La Laguna, pp. 175-185.

Coseriu, E. y Geckeler, H. (1974), «Linguistics and Semantics. Linguistic, especially Functional, Semantics», Sebeok, T. A. (ed.), Current Trends in Linguistics, XII: Linguistics and Adjacent Arts and Sciences, The Hague, Paris, Mouton, pp. 103-171.

Coseriu, E. y Geckeler, H. (1981), Trends in Structural Semantics, Tübingen, Gunter Narr Verlag.

Dubois, J., Giacomo, M., Guespin, L., Marcellesi, C., Marcellesi, J.-B. y Mével, J.-P. (1973), Dictionnaire de linguistique, Paris, Larousse.

Duchácek, O. (1964), «Contribution à l’étude de la sémantique: les synonymes.

Différents types de synonymes», Orbis, 13, pp. 35-49.

Duchácek, O. (1967), Précis de sémantique française, Brno, Universita J. E. Purkyne.

Duchácek, O. (1979), «Synonymie en terminologie», Études romanes de Brno, 10, pp. 9-19.

Ducrot, O. y Todorov, T. (1972), Dictionnaire encyclopédique des sciences du langage, Paris, Éditions du Seuil.

Engler, R. (1968), Lexique de la terminologie saussurienne, Utrecht, Anvers, Spectrum Éditeurs.

Fernández Sevilla, J. (1983), «Sinonimia y polisemia. Implicaciones didácticas», Actas del IV Simposio de Lengua y Literatura Españolas para profesores de bachillerato, Granada, pp. 41-55.

Geckeler, H. (1971), Strukturelle Semantik und Wortfeldtheorie, München, Wilhelm Fink Verlag.

Geckeler, H. (1973), Strukturelle Semantik des Französischen, Tübingen, Max Niemeyer Verlag.

Geckeler, H. (1983), «La semántica, rama clave de la lingüística», Conferencias del trigésimo aniversario de la Universidad de la República, Montevideo, Facultad de Humanidades y Ciencias, pp. 51-65.

Gili Gaya, S. (1964), «El lenguaje de la ciencia y de la técnica», Presente y futuro de la lengua española, 2, Madrid, OFINES, pp.269-276.

Godel, R. (1957), «Lexique de la terminologie», Les sources manuscrites $d u$ 
Cours de linguistique générale de F. de Saussure, Genève, Paris, E. DrozMinard, pp. 252-281.

Greimas, A.-J. (1966), Sémantique structurale. Recherche de méthode, Paris, Larousse.

Greimas, A.-J. y Courtés, J. (1979), Sémiotique. Dictionnaire raisonné de la théorie du langage, Paris, Hachette.

Guiraud, P. (1955), La sémantique, Paris, P.U.F.

Gutiérrez Ordóñez, S. (1981), Lingüística y Semántica (Aproximación funcional), Oviedo, Univ. de Oviedo.

Gutiérrez Ordóñez, S. (1989), Introducción a la semántica funcional, Madrid, Sintesis.

Hartmann, R. R. K. y Stork, F. C. (1972), Dictionary of Language and Linguistics, London, Applied Science Publishers, Ltd.

Heger, K. (1965), «Les bases méthodologiques de l'onomasiologie et du classement par concepts», Travaux de Linguistique et de Littérature, 3,1, pp. 7-32.

Heger, K. (1969a), «La sémantique et la dichotomie de langue et parole», Travaux de Linguistique et de Littérature, 7,1, pp. 47-111.

Heger, K. (1969b), «L'analyse sémantique du signe linguistique», Langue française, 4, pp. 44-66.

Heger, K. (1971), Monem, Wort und Satz, Tübingen, Max Niemeyer Verlag. Heger, K. (1974), Teoría semántica. Hacia una semántica moderna II, Madrid, Alcalá.

Heger, K. (1981), «La semántica lingüística», Lexis, 5,2, pp. 59-93.

Jacobi, D. (1990), «Les séries superordonnées dans les discours de vulgarisation scientifique», Langages, 98, pp. 103-114.

Jacobson, S. (1984), «Three types of terminologies», Hartmann, R. R. K. (ed.), LEXeter' 83 Proceedings. Papers from the International Conference on Lexicography at Exeter, 1983, Tübingen, Niemeyer, pp. 355-361.

Jota, Z. dos S. (1976), Dicionário de lingüística, Rio de Janeiro, Presença.

Kempson, R. (1977), Semantic theory, London, New York, Cambridge University Press. Versión española de R. Cerdà, Barcelona, Teide, 1982.

Kronasser, H. (1952, $2^{\mathrm{a}}$ ed. 1968), Handbuch der Semasiologie. Kurze Einführung in die Geschichte, Problematik und Terminologie der Bedeutungslehre, Heidelberg, Carl Winter Universitätsverlag.

Lázaro Carreter, F. (1974, $3^{\mathrm{a}}$ ed., reimp.), Diccionario de términos filológicos, Madrid, Gredos.

Leech, G. (1974), Semantics, Harmondsworth, Middlesex, Penguin Books Ltd.

Versión española de J. L. Tato G. Espada, Madrid, Alianza, 1977.

Lerat, P. (1990), «L'hyperonymie dans la structuration des terminologies», Langages, 98, pp. 79-86.

Lewandowski, T. (1982), Diccionario de lingüística, traducción de Mํㅡ. Larcía- 
Denche Navarro y E. Bernárdez, Madrid, Cátedra.

Lyons, J. (1968), Introduction to theoretical linguistics, Cambridge, New York,

New Rochelle, Melbourne, Sidney, Cambridge University Press. Versión

española de R. Cerdà, Barcelona, Teide, 1975, $3^{\underline{a}} \mathrm{ed}$.

Lyons, J. (1977), Semantics, 2 vols., Cambridge, London, New York, Melbourne,

Cambridge University Press. Versión castellana de R. Cerdà, Barcelona, Teide, 1980.

Lyons, J. (1981), Language, meaning and context, Bungay, Suffolk, Fontana

Paperbacks. Traducción de S. Alcoba, Barcelona, Buenos Aires, Paidós, 1983.

Marouzeau, J. (1951, 3ª ed.), Lexique de la terminologie linguistique français, allemand, anglais, italien, Paris, Librairie Orientaliste Paul Geuthner.

Martinet, A. (1967, 2ª ed.), Éléments de linguistique générale, Paris, Librairie Armand Colin.

Martinet, A. (dir.) (1972), La lingǘstica. Guía alfabética, traducción de C. Manzano, Barcelona, Anagrama.

Martínez Hernández, M. (1984), «Estado actual de la semántica y su aplicación al griego antiguo», Martínez Díez, A. (ed.), Actualización científica en Filología griega, Madrid, I.C.E. Univ. Complutense, pp. 355-413.

Mounin, G. (comp.) (1974), Dictionnaire de la linguistique, Paris, P.U.F.

Muñoz Núñez, M를 D. (1993), El problema semántico de la polisemia léxica, Tesis de licenciatura, Univ. de Cádiz.

Muñoz Valle, I. (1975), «En torno a la sinonimia. (¿Existen los sinónimos perfectos?)», Durius, 3,6, pp. 263-289.

Ogden, C. K. y Richards, I. A. (1960, 10ª ed.), The meaning of meaning. A study of the influence of language upon thought and of the science of symbolism, London, Routledge \& Kegan Paul Ltd.

Pottier, B. (ed.) (1973), Le langage, Paris, Denoël.

Read, A. W. (1948), «An account of the word 'Semantics'», Word, 4, pp. 78-97. Reisig, C. K. (1839), «Semasiologie oder Bedeutungslehre», Haase, F. (ed.), Professor K. Reisig's Vorlesungen über lateinische Sprachwissenschaft, Leipzig, pp. 286-307.

Salvador, G. (1985), Semántica y lexicología del español, Madrid, Paraninfo. Saussure, F. de (1922, 2ª ed.), Cours de linguistique générale. Publié par C. Bally et A. Sechehaye avec la collaboration de A. Riedlinger, Paris, Payot.

Schick, C. (1960), Il linguaggio, Torino.

Schippan, T. (1972, 2ª ed. 1975), Einführung in die Semasiologie, Leipzig.

Söll, L. (1966), Synonymie und Bedeutungsgleichheit», Germanisch-Romanische Monatschrift, 47,16, pp. 90-99.

Spillner, B. (1992), «Konzeption und Definition von 'Strukturalismus' in der Linguistik», Anschütz, S. R. (ed.), Texte, Sätze, Wörter und Moneme. 
Festschrift für Klaus Heger zum 65. Geburtstag, Heidelberg, Heidelberger Orientverlag, pp. 607-624.

Stern, G. (1931), Meaning and Change of Meaning. With Special Reference to the English Language, Göteborg, Göteborgs Högskolas Arsskrift XXXVIII. Struck, E. (1940, $2^{\mathrm{a}}$ ed. 1954), Bedeutungslehre. Grundzüge einer lateinischen und griechischen Semasiologie, Leipzig, Berlin (2a ed. Stuttgart).

Swiggers, P. (1983), «Sémasiologie et onomasiologie: opposition, recouvrement et complémentarité», Angelet, C., Melis, L., Mertens, F. J. y Musarra, F. (eds.), Langue, dialecte, littérature. Études romanes à la mémoire de Hugo Plomteux, Leuven, pp. 431-438.

Todorov, T. (1975), «Signifiance et sens», Mélanges linguistiques offerts à Émile Benveniste, Paris, pp. 509-515.

Trujillo, R. (1972), «Gramática, lexicología y semántica», Revista Española de Lingüística, 2,1, pp. 103-109.

Trujillo, R. (1974), «El lenguaje de la técnica», Doce ensayos sobre el lenguaje, Madrid, Rioduero, pp. 197-211.

Trujillo, R. (1983a), «La semántica», Abad, F. y García Berrio, A. (coords.), Introducción a la lingüística, Madrid, Alhambra, pp. 185-215.

Trujillo, R. (1983b), «El signo: ¿Cosa que se pone en lugar de otra?», Serta Philologica F. Lázaro Carreter, 1, Madrid, pp. 613-623.

Trujillo, R. (1988), Introducción a la semántica española, Madrid, Arco/Libros. Ullmann, S. (1952), Précis de sémantique française, Berne, Éditions A. Francke S.A.

Ullmann, S. (1956), «The concept of meaning in linguistics», Archivum Linguisticum, 8,1, pp. 12-20.

Ullmann, S. (1957, $2^{\mathrm{a}}$ ed.), The Principles of Semantics, Oxford, Basil Blackwell. Ullmann, S. (1964a), Semantics. An Introduction to the Science of Meaning, Oxford, Basil Blackwell.

Ullmann, S. (1964b), Language and style, Oxford, Basil Blackwell.

Ullmann, S. (1973), Meaning and style, Oxford, Basil Blackwell.

Ullmann, S. (1974, reimp.), Introducción a la semántica francesa. Traducción y anotación por E. de Bustos Tovar, Madrid, C.S.I.C.

Welte, W. (1985), Lingüística moderna. Terminología y bibliografía. Versión española de F. Meno Blanco, Madrid, Gredos.

Wolf, L. (1974), «Zur Diskussion über Terminologie und Semantik», Kapp, V. (ed.), Übersetzer und Dolmetscher. Theoretische Grundlagen, Ausbildung, Berufspraxis, Heidelberg, pp. 50-61.

Wunderli, P. (1992), «»Phonétique» und «Phonologie». Zur Problematik der linguistischen Terminologie», Anschütz, S. R. (ed.), Texte, Sätze, Wörter und Moneme. Festschrift für Klaus Heger zum 65. Geburtstag, Heidelberg, Heidelberger Orientverlag, pp. 693-718.

Zvegincev, V. A. (1957), Semasiologija, Moskva. 